\title{
A Note on Recent Trends in Suicide in Fiji
}

\author{
Peter M. Forster \\ University of Gloucestershire, United Kingdom
}

\author{
Selina C. Kuruleca \\ University of the South Pacific, Fiji \\ C.R. Auxier \\ Capella University, United States of America
}

\begin{abstract}
$T_{\text {tion }}^{\text {hat }}$ he incidence of suicides and attempted suicides is one indicator of mental health and life satisfaction within the population. The trends in suicidal behaviour vary with sex and ethnicity within the population, and underscore the impact of culture on people's lives and wellbeing. Although incidence of suicide provides important information, there has been no systematic reporting of data within the country. The only source of national data is the statistics unit of Fiji's police force, who record all deaths that were not caused by disease, along with such data as the sex and ethnicity of the deceased. The police are willing to provide information on suicide to researchers and government bodies, within the constraints of having the staff and other resources needed to provide such information. The present article explores what we know about this important social issue, and investigates some potential ways forward for dealing with it at individual and group levels.
\end{abstract}

Auxier, Forster and Kuruleca (2005) identified social factors that are driving large-scale changes in Fiji. The lingering effects of colonisation and pressures of globalisation were two of the more significant. They also reported the widely held belief that social problems will increase in the next 5 years due to the effects of Fiji's economic decline and urban drift. Although official figures for recent years are not yet available, unemployment rates are probably high and rising. Figures available at the time of writing show an increase from $7.6 \%$ to $12.1 \%$ from 1999 to 2000 (Fiji, 2005). Many people have been moving from predominantly rural areas to the main cities of Suva, Nadi and Lautoka in search of jobs and economic stability. The two most mobile groups have been Indian agricultural workers and young Indigenous Fijians. The Indian agricultural workers were often forced to move because of expired sugar cane land leases. When the leases expired and were not renewed, the workers simultaneously lost their livelihood and their homes. Because of a coincidence in the dates of onset of these leases, an increase in lease expiries took place in the years between 1995 and the present. There have also been movements of mainly young, Indigenous Fijians looking for work and alternatives to a rural, agricultural lifestyle. Towns and cities have become burdened with displaced and disenfranchised people with few resources. These people have moved from being accepted members of well-ordered rural communities to the chaotic and impersonal atmosphere of urban life. The response of some has been an increase in domestic violence, crimes against property, child abuse, strains in family relationships and suicide. Support for the significant role played by social disintegration as a result of urban drift is provided by Forsyth (1980), who observed higher levels of marital conflict among urban dwellers in the Lae province of Papua New Guinea than among those living in their traditional communities.

\section{Suicide Rates in Fiji}

Prior to the present study, suicide data were collected by Adinkrah and reported in 1995, and by Booth, reported in 1997 and 1999. For the population as a whole, the standardised annual rate of suicide for 2002 was 15 per 100,000 population for males and 11 for females. However, there are considerable differences between the rates for the different ethnic groups in Fiji. In the same year, the rate for Indigenous Fijians (51\% of the population) was four, while that for Fiji Indians ( $44 \%$ of the population) was 
24. There are no separate figures for the remaining ethnic groups, which mainly consist of Chinese, Europeans and people of other Pacific island nations, but as a whole this mixed group had a standardised suicide rate of two. At the time of writing, figures for interactions between sex and ethnicity were not available.

Booth (1997) reported that the Pacific region had some of the highest youth suicide rates in the world. Suicide rates for young males in the Federated States of Micronesia, Marshall Islands, Guam, Palau, Samoa and among Fiji Indians (with a standardised rate of 57) were among the highest anywhere. The same was true for young females in Samoa and again among Fiji Indians (with a standardised rate of 60). Booth reported that in these countries suicide was the highest cause of death for the 15-24-years age group. These rates were derived from police figures, which did not include drowning or some apparent accidents because of the uncertainty of intent. Consequently in Fiji, as elsewhere, the official figures probably underestimate the actual number of suicides. The most commonly used methods were hanging and ingestion of weed killer.

\section{Causes}

Explanations of these high rates that are accepted in other parts of the Pacific cannot entirely account for the ethnic differences in Fiji, or the unusually high levels for younger females. A widely accepted reason for high levels in other parts of the Pacific is social change resulting from developmental pressures. This leads to intergenerational conflicts, which are assumed to weigh heavier on young people (Rubinstein, 1992) and add to the factors identified at the start of this article.

Booth (1999) proposed that the relatively high level of suicide by young Fiji Indian females is the result of the greater changes they have experienced in recent years, compared to young Fiji Indian males and older Fiji Indians. They have been changing their roles in marital relationships so they derive less power from traditional female sources of power, such as son-bearing, and more from traditionally male sources such as socioeconomic achievement. They marry later, pursue education longer and engage more in paid employment than previously. Fiji Indian males are assumed to have gone through fewer changes in the same period. Differential pressures of these kinds are consistent with accounts of the experience of young Asian females living within a variety of other cultures, as discussed in Ghuman (2005).

Booth (1999) reported that marriage and parenthood, in the Pacific region as elsewhere, are protective against suicide. However, she reported that this appears not to be true for young Fiji Indian females. Booth proposed that the high rate of arranged marriages leads to disharmony with husband and/or in-laws in the early years of marriage and reduces the protective effect of marriage. Childlessness is also identified as a source of tension within marriages. This is supported by a study by Merrill and Owens (1986) of female Indian migrants into the United Kingdom who had attempted suicide. They found that arranged marriages, rejection of arranged marriages and associated marital problems were reported as the main contributory factors. Taken together, these factors provide a speculative, but plausible account of the gender/age/ethnicity interaction in suicide rates among young Fiji Indians. This picture is complicated, however, by Haynes' (1984) survey of police and medical records on the island of Vanua Levu between 1979 and 1982. She reported higher rates of suicide among females under the age of 30 and also among older males. Older males have relatively higher status in this community, so Booth's (1999) account provides only a partial explanation for sex differences in suicide rates.

Anecdotal evidence, mainly derived from newspaper reports, suggests that suicide by young people increases after school examination results are released, specifically among those who failed examinations. These reports almost entirely involve Fiji Indians. Discussions with members of the two main ethnic groups, particularly secondary and tertiary teachers, suggest that parents from these groups place differential value on educational success. It is widely accepted that Fiji Indians value formal education and educational success more highly than Indigenous Fijians. These accounts propose that Fiji Indian parents put more pressure on their children to succeed in school and that some young people are unable to cope with educational failure.

There are also differences in the suicide rate for the various ethno-religious groups within the Indian population. In the 1986 census, Indians described themselves as 79\% Hindu, 16\% Muslim and 5\% Christian (Fiji, 1988). However, in 1989/90, suicides were distributed as $91 \%$ Hindu, 7\% Muslim and 2\% Christian. This pattern is consistent with a model in which suicide is more or less likely, depending on one's culture. Cultures in which suicide is taboo (Christian and Muslim) exert an inhibitory effect, while cultures in which it is tolerated (Hindu) exert a facilitating effect. It is possible to read accounts of suicide in Hindu scriptures such as the Upanishads and Dharmasutras, although they distinguish between religious and secular suicide, with secular suicide not being condoned. Suicide has been accepted as a form of political protest in India and for warriors facing defeat. Although it is now illegal, the traditional Indian practice of sati, or self-immolation by a widow on her husband's funeral pyre, was an acceptable form of suicide for centuries. It is important for health-care workers in Fiji to be aware of such ethno-religious factors.

International comparisons are difficult because of differential reporting. Evidence from elsewhere (New South Wales Health Department, 2000) suggests 
that the suicide levels of immigrant groups are more like those of the former culture than the new culture. However, this may apply more to older migrants than to younger migrants.

Speculatively, it is possible that the recreational drinks used by the two main ethnic groups may have a part to play in the different suicide rates. Fijians are more likely to drink kava (or yaqona as it is called locally) than alcoholic drinks, whereas Fiji Indians are more likely to drink alcoholic drinks. Kava is a relaxant that makes impulsive behaviour less likely. Traditionally, chiefs drank it before important occasions such as signing treaties and it made impulsive conflict less likely. Those who drink alcohol are more impulsive and are at increased risk of suicide, so this may be an additional contributory factor.

\section{Intervention}

The differences between the various ethnic groups in suicide profiles provide a challenge to health workers in Fiji, including counsellors and social workers. It is unusual for health workers to deal exclusively with clients of the same ethnicity as themselves. They need to be sensitive to the differences in presenting issues, in attitudes about self-harm and to outcomes that are considered acceptable, when working with clients of different ethnicity.

Suicide data also question interpreting suicide as exclusively a mental health issue. Fiji experienced the overthrow of democratically elected governments in 1987 and 2000. In each case, ethnicity was a factor as these were governments containing a higher proportion of Fiji Indians than Indigenous Fijians. In those years and the following year, suicides by Fiji Indians increased, while those for other ethnic groups did not. Whether this increase is real or coincidental could only be determined if more detailed information is collected and analysed more systematically than is currently possible.

The growing public awareness of suicide in general, and of suicide by young people in particular, has led to discussion at cabinet level, public debate, the establishment of the National Committee On the Prevention of Suicide (NCOPS) in 2001 and calls by groups such as teachers and non-governmental organisations (NGOs) for more trained counsellors. It is one of the factors most likely to have led to the growth in enrolments in counselling programs at the regional university, The University of the South Pacific (USP), since their launch in 1997.

NCOPS has launched a number of important initiatives, which have been given the highest backing from the national government, government ministries, the regional university and other significant community groups. These include creating a program to participate in World Suicide Prevention Day, launching nationwide secondary school initiatives with the training of senior student peer educators and contacts in each school, distributing educational materials to secondary schools, creating radio talkback programs in the three main languages spoken in Fiji (Fijian, Hindi and English), helping the Media Council of Fiji update its code of ethics to include the ethics of suicide reporting, making presentations and recommendations to a Paraquat seminar and helping to set up a telephone crisis line in collaboration with the Fiji Association of Social Workers.

Dialogue between the National Committee on the Prevention of Suicide (NCOPS) and Fiji's Ministry of Education has resulted in plans to increase both the numbers and types of counselling services available to young people, particularly secondary school students. Such schemes are praiseworthy because they will provide support and help to students facing a wide range of difficulties, in addition to those with suicidal ideation or intent. The Ministry of Education has recognised the problem, and is discussing options for independent, therapeutically oriented counsellors to be available to students. Those of us who are responsible for those programs are now being challenged to help improve people's quality of life and reduce the number of suicides, particularly by young people, in Fiji.

In 2003, NCOPS organised a symposium on suicide prevention in Fiji where a national strategy on suicide prevention was developed. Implementation of this strategy is underway, with the support of funding from the government of Fiji. If funding from more sources becomes available then this momentum can be maintained.

Finally, given that suicide is such a significant issue, particularly for young people in Fiji and elsewhere in the region, the authors think that it is vitally important that a permanent watch be kept on suicide data, with detailed summaries produced every year. This is important to evaluate the success of efforts by NCOPS and others. It would also allow for more detailed study of factors described above and elsewhere, such as the part played by religion, age, geographical location, sex and culture in suicide. Such studies are needed both to understand the problem and to target limited resources where they can do the most good. Bodies such as the Department of Statistics, the Ministry of Health or the University of the South Pacific are among the few with the resources and skills necessary to sustain such a data-gathering exercise. We commend it for their urgent attention.

\section{References}

Adinkrah, M. (1995). Crime, deviancy, and delinquency in Fiji. Suva: Fiji Council of Social Services.

Auxier, C.R., Forster, P.M. \& Kuruleca, S. (2005). Counselling in Fiji. Journal of Mental Health Counseling, 27(2), 142-148. 
Booth, H. (1998). Casualties of development? Child and youth suicide in the Pacific. Development Bulletin on ChildFocused Development, 44, 48-50.

Booth, H. (1999). Gender, power and social change: Youth suicide among Fiji Indians and Western Samoans. The Journal of the Polynesian Society, 108, 39-68.

Fiji Islands Department of Statistics (1988). Report on the Fiji population census 1986 (Vol. 1). Suva, Fiji: Author.

Fiji Islands Bureau of Statistics (2005). Fiji facts and figures. Suva, Fiji: Author.

Forsyth, S.J. (1980, November.). [Problem behaviours of Lae residents]. Unpublished notes.
Ghuman, P.S. (2005). Daughters of traditions. The Psychologist, $18,620-622$.

Haynes, R.H. (1984). Suicide in Fiji: a preliminary study. The British Journal of Psychiatry, 145, 433-438.

Merrill, J., \& Owens, J. (1986) Ethnic differences in selfpoisoning. British Journal of Psychiatry, 148, 708-712.

New South Wales Health Department, Sydney. (2000). Suicide in New South Wales: We need to know more. State Health Publication 990208. Sydney, Australia.

Rubinstein, D. (1992). Suicidal behaviour in Micronesia. In K.L. Peng \& W.S. Tseng (Eds.), Suicidal behaviour in the Asia-Pacific region (pp. 199-229). Singapore: Singapore University Press. 